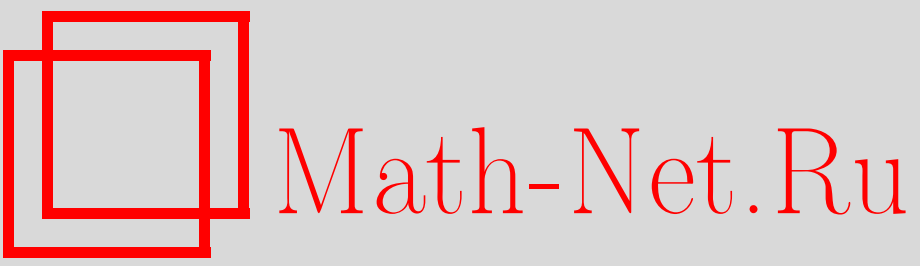

Е. О. Бурлаков, Т. В. Жуковская, Е. С. Жуковский, Н. П. Пучков, О непрерывных и разрывных моделях нейронных полей, Итоги науки и техн. Сер. Соврем. мат. и ее прил. Темат. обз., 2019, том 165, 10-20

DOI: https://doi.org/10.36535/0233-6723-2019-165-10-20

Использование Общероссийского математического портала Math-Net.Ru подразумевает, что вы прочитали и согласны с пользовательским соглашением

http: //www.mathnet.ru/rus/agreement

Параметры загрузки:

IP: 52.87 .193 .239

26 апреля 2023 г., 13:18:08 


\title{
О НЕПРЕРЫВНЫХ И РАЗРЫВНЫХ МОДЕЛЯХ НЕЙРОННЫХ ПОЛЕЙ
}

\author{
() 2019 г. Е. О. БУРЛАКОВ, Т. В. ЖУКОВСКАЯ, \\ Е. С. ЖУКОВСКИЙ, Н. П. ПУЧКОВ
}

\begin{abstract}
АннотАция. Статья посвящена исследованиям в математической нейробиологии, целью которых являлось установление связи между подходами к моделированию нейронных полей, основанными на непрерывных и разрывных моделирующих уравнениях. Приводится обзор работ по данной тематике и предлагается новый аппарат решения подобных задач, основанный на абстрактных включениях Вольтерры и позволяющий обобщить ряд полученных ранее результатов.
\end{abstract}

Ключевъе слова: математическая модель, нейронное поле, интегральное уравнение, уравнение Гаммерштейна, разрешимость, непрерывная зависимость от параметров.

\section{ON CONTINUOUS AND DISCONTINUOUS MODELS OF NEURAL FIELDS}

\author{
(c) 2019 E. O. BURLAKOV, T. V. ZHUKOVSKAYA, \\ E. S. ZHUKOVSKIY, N. P. PUCHKOV
}

\begin{abstract}
This paper is devoted to research in mathematical neurobiology whose purpose is the establishment of a connection between approaches to the modeling of neural fields based on continuous and discontinuous equations. We review works on this topic and propose a new method for solving such problems based on Volterra's abstract inclusions, which allows one to generalize some previously obtained results.
\end{abstract}

Keywords and phrases: mathematical model, neural field, integral equation, Hammerstein equation, solvability, continuous dependence on parameters.

AMS Subject Classification: 47J05, 47H04, 35R09, 45K05, 47H30, 92 F99

1. Введение. В течение последних десятилетий существенно возрос интерес к математическим моделям электрической активности коры головного мозга. Первые подобные модели восходят к работам H. R. Wilson, J. D. Cowan, S. Amari. Дальнейшие исследования в этой области позволили дать математические описания нормального функционирования головного мозга (например, при обработке визуальных стимулов - исследования H. Sompolinsky и R. Shapley, при управлении движениями - исследования J. S. Taube и J. P. Bassett, при обращении к памяти -

Работа Е. О. Бурлакова выполнена при поддержке Российского фонда фундаментальных исследований (проекты № 17-41-680975, № 18-31-00227) и фонда развития теоретической физики и математики «Базис» (проект № 18-1-7-37-1).

Работа Е. С. Жуковского выполнена при поддержке Российского фонда фундаментальных исследований (проекты № 17-01-00553, № 17-41-680975, № 17-51-12064) и Министерства образования и науки Российской Федерации (государственное задание № 3.8515.2017/БЧ).. 
исследования J. M. Fuster, G. Alexander, X.-J. Wang), а также математические описания различных нарушений его нормальной активности (например, при болезни Паркинсона - исследования S. J. Schiff, во время приступа эпилепсии - исследования F. H. Lopes Da Silva, P. Taylor).

Наиболее известной моделью, описывающей электрическую активность в коре головного мозга, является так называемая модель Amari (см. [2]):

$$
\frac{\partial u(t, x)}{\partial t}=-\alpha u(t, x)+\int_{\Omega} \omega(x-y) f(u(t, y)) d y, \quad t \geqslant 0, \quad x \in \Omega \subseteq \mathbb{R}^{m}, \quad \alpha>0 .
$$

Здесь функция $u(t, x)$ характеризует активность нейрона в позиции $x$ в момент времени $t$, функции активации $f(u)$ - степень влияния нейрона с активностью $u$ на другие нейроны, функция связи $\omega$-силу взаимодействия между нейронами. Исходя из биофизических оснований, входящие в уравнение (1) функции являются непрерывными, а зачастую даже гладкими: функция $f$ типично имеет сигмоидальную форму, $\omega$ - форму гауссовой функции.

Для случая непрерывной функции активации $f$ разрешимость и корректная разрешимость в различных функциональных пространствах модели Amari и ее обобщений были показаны в $[3,4]$. Использование в таких моделях разрывной функции типа Хевисайда вместо непрерывной функции $f$ позволяет существенно упростить их численное исследование, а также получить в явном виде многие важные с точки зрения нейробиологии типы решений. «Обоснованием» такой замены обычно служит трактовка функции Хевисайда в качестве предела сигмоидальных функций нарастающей крутизны (т.е. при стремлении к нулю промежутка между «активацией» и «насыщением»). В последние годы появились исследования, дающие строгие обоснования возможности такого перехода (в основном для определенных типов решений уравнения (1)), использующие теории топологической степени, частично упорядоченных пространств, компактность в специальных топологиях и другие методы нелинейного функционального анализа.

Данная работа построена следующим образом. Раздел 2 начинается с введения необходимых обозначений; п. 2.1 содержит описания результатов вышеупомянутых исследований, полученных для стационарных пространственно локализованных решений уравнения (1), отвечающих нормальной функции головного мозга (см. [1]) и, соответственно, занимающих особенно важное место в исследованиях математической нейробиологии. В п. 2.2 освещается исследование близости нестационарных решений уравнения (1) с разрывной и непрерывной функцией активации, а также предлагается метод решения данной задачи для обобщения уравнения (1), учитывающего эффекты памяти и запаздывания, основанный на теории включений Вольтерры. В разделе 3 обсуждаются приведенные результаты и возможные перспективы дальнейших исследований.

2. Основные результаты. Для удобства дальнейшего изложения введем следующие обозначения. Пусть $\mathbb{R}^{m}-m$-мерное вещественное векторное пространство с нормой $|\cdot|$. Для любого измеримого по Лебегу множества $D \subset \mathbb{R}^{m}$ обозначим через $\operatorname{mes}(D)$ его меру Лебега.

Пусть $\mathcal{B}$ - некоторое банахово пространство с нормой $\|\cdot\|_{\mathcal{B}}$. Обозначим через $\overline{\operatorname{conv}}(\mathcal{B})$ множество всех непустых выпуклых замкнутых подмножеств $\mathcal{B}$.

Для любых $M \subset \mathcal{B}, r>0$ определим множество

$$
B_{\mathcal{B}}(M, r)=\left\{b \in \mathcal{B},\|b-m\|_{\mathcal{B}}<r, m \in M\right\}
$$

и обозначим через $\bar{M}$ замыкание $M$ в $\mathcal{B}$.

Пусть $\Omega$-замкнутое подмножество $\mathbb{R}^{m}, \Omega_{r}=\Omega \cap \overline{B_{\mathbb{R}^{m}}(0, r)}, T>0$. Для любых $r>0$ и $k=0,1,2, \ldots$ обозначим через $C^{k}\left(\Omega_{r}, \mathbb{R}^{n}\right)$ пространство всех функций $\xi: \Omega_{r} \rightarrow \mathbb{R}^{n}$, непрерывных вместе с первыми $k$ производными $\xi^{(i)}, i=1, \ldots, k$, с нормой

$$
\|\xi\|_{C^{k}\left(\Omega_{r}, \mathbb{R}^{n}\right)}=\sum_{i=0}^{k} \max _{x \in \Omega_{r}}\left|\xi^{(i)}(x)\right|, \quad \xi^{(0)}=\xi ;
$$

через $B C\left(\Omega, \mathbb{R}^{n}\right)$ - пространство всех непрерывных ограниченных функций $\zeta: \Omega \rightarrow \mathbb{R}^{n}$ с нормой

$$
\|\zeta\|_{B C\left(\Omega, \mathbb{R}^{n}\right)}=\sup _{x \in \Omega}|\zeta(x)| .
$$


Будем использовать стандартное обозначение $C\left(\Omega_{r}, \mathbb{R}^{n}\right)$ в случае $k=0$. Обозначим через $L\left(\Omega, \mathbb{R}^{n}\right)$ пространство всех измеримых суммируемых функций $\eta: \Omega \rightarrow \mathbb{R}^{n}$ с нормой

$$
\|\eta\|_{L\left(\Omega, \mathbb{R}^{n}\right)}=\int_{\Omega}|\eta(x)| d x .
$$

Пусть $C_{0}\left([0, T] \times \Omega, \mathbb{R}^{n}\right)$ - пространство непрерывных функций $\nu:[0, T] \times \Omega \rightarrow \mathbb{R}^{n}$, удовлетворяющих в случае неограниченности множества $\Omega$ дополнительному условию

$$
\lim _{|x| \rightarrow \infty} \nu(t, x)=0 \quad \forall t \in[0, T]
$$

с нормой

$$
\|\nu\|_{C_{0}\left([0, T] \times \Omega, \mathbb{R}^{n}\right)}=\max _{(t, x) \in[0, T] \times \Omega}|\nu(t, x)| .
$$

Обозначим через $C_{0}\left([0, \infty) \times \Omega, \mathbb{R}^{n}\right)$ локально выпуклое пространство функций $\widehat{\nu}:[0, \infty) \times \Omega \rightarrow \mathbb{R}^{n}$, удовлетворяющих в случае неограниченности множества $\Omega$ дополнительному условию

$$
\lim _{|x| \rightarrow \infty} \widehat{\nu}(t, x)=0 \quad \forall t \geqslant 0
$$

с топологией равномерной сходимости на множествах вида $[0, T] \times \Omega, 0<T<\infty$.

2.1. Стачионарные локализованные решения уравнения нейронного поля. В [7] исследовались стационарные пространственно локализованные решения уравнения (1) в случае $\Omega=\mathbb{R}$. Ниже излагаются результаты этой работы.

В случае, если стационарное решение (1) существует, оно удовлетворяет следующему уравнению:

$$
u(x)=\int_{\mathbb{R}} \omega(x-y) f(u(y)) d y, \quad x \in \mathbb{R} .
$$

Относительно функции связи $\omega$ предполагается, что выполнено следующее условие:

$\left(\mathbf{A}_{\omega}^{1}\right) \quad \omega(x)=\omega(-x)$ для всех $x \in \mathbb{R} ; \omega \in B C(\mathbb{R}, \mathbb{R}) \cap L(\mathbb{R}, \mathbb{R})$; существует такое $C>0$, что $\left|\omega^{\prime}(x)\right| \leqslant C$ для всех $x \in \mathbb{R}$.

С учетом замены $r(x, y)=\omega(x-y)+\omega(x+y)$ уравнение $(2)$ принимает вид

$$
u(x)=\int_{0}^{\infty} r(x, y) f(u(y)) d y, \quad x \in \mathbb{R} .
$$

Пусть задано $\theta>0$. Будем говорить, что $U(x)$ - пространственно локализованное стационарное симметричное решение $(1)$ ширины $A(A>0)$, если для некоторого связного множества $\Theta \subset \mathbb{R}$, $\operatorname{mes}(\Theta)=A$, неравенство $U(x) \geqslant \theta$ выполнено тогда и только тогда, когда $x \in \Theta$.

В случае, если $f$ - функция вида

$$
f_{0}(u)= \begin{cases}0, & u \leqslant \theta \\ 1, & u>\theta\end{cases}
$$

то пространственно локализованное стационарное симметричное решение (1) принимает вид

$$
U(x)=\int_{\Theta} \omega(x-y) d y, \quad x \in \mathbb{R} .
$$

Учитывая свойство инвариантности уравнения (2) относительно сдвигов (для любого решения $U(x)$ уравнения (2) функция $U(x+\widehat{x})$ также является решением $(2)$ при любом $\widehat{x} \in \mathbb{R})$, можем записать последнее равенство в виде

$$
U(x)=\int_{-A / 2}^{A / 2} \omega(x-y) d y \quad \text { или } \quad U(x)=\int_{0}^{A / 2} r(x, y) d y, \quad x \in \mathbb{R} .
$$


Пусть для любого $h>0$ заданы такая неубывающая функция $\widehat{f} \in C(\mathbb{R},[0,1])$, что $\widehat{f}(u)=0$, $u \leqslant \theta$ и $\widehat{f}(u)=1, u \geqslant \theta+h$ и функция $f_{h} \in C(\mathbb{R},[0,1])$,

$$
f_{h}(u)= \begin{cases}0, & u<\theta+h, \\ 1, & u \geqslant \theta+h .\end{cases}
$$

Для любого $u \in \mathbb{R}$, очевидно, имеем

$$
f_{h}(u) \leqslant \widehat{f}(u) \leqslant f_{0}(u) .
$$

Предположение 2.1. Предположим, что существуют пространственно локализованные стационарные симметричные решения $U_{0}$ ширины $2 A_{0}$ и $U_{h}$ ширины $2 A_{h}$ уравнения (1) при $f=f_{0}$ и $f=f_{h}$, соответственно, причем $A_{h}<A_{0}$.

Предположение 2.2. Предположим, что при всех $x, y \in\left[A_{h}, A_{0}\right]$ имеет место неравенство $r(x, y) \geqslant 0$.

Всюду далее для случая, когда $f=\widehat{f}$, рассматриваются пространственно локализованные стационарные симметричные решения $\widehat{U}$ уравнения (1), для которых выполнены следующие условия:

$$
U_{h}(x)>h \Rightarrow \widehat{U}(x)>\theta+h, \quad U_{0}(x)<0 \Rightarrow \widehat{U}(x)<\theta .
$$

Такие решения $\widehat{U}$ при всех $x \in\left[A_{h}, A_{0}\right]$ удовлетворяют уравнению

$$
U(x)=U_{h}(x)+\int_{A_{h}}^{A_{0}} r(x, y) \widehat{f}(U(y)) d y
$$

и являются элементами конусного отрезка

$$
\left[U_{h}, U_{0}\right]=\left\{u \in C\left(\left[A_{h}, A_{0}\right], \mathbb{R}\right), \quad U_{h}(x) \leqslant u(x) \leqslant U_{0}(x), \quad x \in\left[A_{h}, A_{0}\right]\right\} .
$$

При помощи теории монотонных операторов в частично упорядоченных пространствах в [7] получено следующее утверждение.

Теорема 2.1. Пусть выполнено условие $\left(\mathbf{A}_{\omega}^{\mathbf{1}}\right)$, а такэе предположения 2.1 и 2.2. Тогда уравнение (4) имеет решение. Кроме того, для итерачий $\mathcal{F}^{i}$ оператора

$$
\begin{gathered}
\mathcal{F}:\left[U_{h}, U_{0}\right] \rightarrow C\left(\left[A_{h}, A_{0}\right], \mathbb{R}\right), \\
(\mathcal{F} U)(x)=U_{h}(x)+\int_{A_{h}}^{A_{0}} r(x, y) \widehat{f}(U(y)) d y, \quad x \in\left[A_{h}, A_{0}\right],
\end{gathered}
$$

имеют место соотношения

$$
\left\|\mathcal{F}^{i} U_{0}-U^{*}\right\|_{C\left(\left[A_{h}, A_{0}\right], \mathbb{R}\right)} \rightarrow 0, \quad\left\|\mathcal{F}^{i} U_{h}-U_{*}\right\|_{C\left(\left[A_{h}, A_{0}\right], \mathbb{R}\right)} \rightarrow 0 \quad \text { npu } i \rightarrow \infty,
$$

где $U_{*}$ и $U^{*}-$ такие решения уравнения (4), что для любого решения $U \in\left[U_{h}, U_{0}\right]$ уравнения (4) и любого $x \in\left[A_{h}, A_{0}\right]$ выполнено неравенство $U_{*}(x) \leqslant U(x) \leqslant U^{*}(x)$.

Следствие 2.1. Пусть выполнены условия теоремы 2.1. Если $U^{*}=U_{*}=\widehat{U}$, то $\widehat{U}$ является единственным решением уравнения (4) в множестве $\left[U_{h}, U_{0}\right]$.

На основе данных утверждений авторами работы [7] были предложены два численных метода построения пространственно локализованного стационарного симметричного решения для непрерывной функции активации $f=\widehat{f}$ : с помощью итераций по ширине такого решения и «прямыми итерациями» (т.е. по схеме теоремы 2.1).

В [7] также был решен вопрос о возможности продолжения решения $\widehat{U} \in C\left(\left[A_{h}, A_{0}\right], \mathbb{R}\right)$ до такого пространственно локализованного стационарного симметричного решения $\widehat{U} \in C(\mathbb{R}, \mathbb{R})$, что

$$
\begin{array}{ll}
\widehat{U}(x)>\theta+h, & x \in\left[0, A_{h}\right), \\
\widehat{U}(x)<\theta, & x \in\left(A_{0}, \infty\right) .
\end{array}
$$


Используя теорему 2.1, при дополнительных предположениях можно получить результат о близости исследуемых решений при переходе от разрывной к непрерывной функции активации. Сформулируем соответствующие предположение и утверждение.

Предположение 2.3. Пусть для заданного $\theta>0$ найдется такое $\widehat{h}>0$, что при любом $h$, $0<h \leqslant \widehat{h}$, существуют локализованные стационарные симметричные решения $U_{0}$ ширины $2 A_{0}$ и $U_{h}$ ширины $2 A_{h}$ уравнения (1) при $f=f_{0}$ и $f=f_{h}$, соответственно, причем $A_{h}<A_{0}$.

Следствие 2.2. Пусть выполнено условие $\left(\mathbf{A}_{\omega}^{1}\right)$, а также предположения 2.2 и 2.3. Тогда уравнение (4) имеет решение $U(x)$. Кроме того, при $h \rightarrow 0$ имеют место соотношения

$$
\left\{\begin{array}{l}
U_{h}(x) \leqslant U(x) \leqslant U_{0}(x), \\
U_{h}(x) \rightarrow U_{0}(x)
\end{array} \forall x \in\left[A_{\widehat{h}}, A_{0}\right] .\right.
$$

В [8] была показана непрерывная зависимость локализованного стационарного решения (1) $($ в случае $\Omega=\mathbb{R})$ при переходе от гладкой функции активации к разрывной функции типа Хевисайда. Этот результат был обобщен в [3] на случай $m$-мерной усредненной модели Amari:

$$
\begin{gathered}
\frac{\partial u\left(t, x, x_{f}\right)}{\partial t}=-u\left(t, x, x_{f}\right)+\int_{\Omega} \int_{\mathcal{Y}} \omega\left(x-y, x_{f}-y_{f}\right) f(u(t, y)) d y_{f} d y, \\
t>0, \quad x \in \Omega \subseteq \mathbb{R}^{m}, \quad x_{f} \in \mathcal{Y} \subset \mathbb{R}^{k}
\end{gathered}
$$

полученной из уравнения (1) путем введения периодической микроструктуры и последующего усреднения (подробнее см. [4] и приведенную там библиографию). В качестве элементарной единицы микроструктуры был выбран $k$-мерный тор $\mathcal{Y}$. Ниже приводятся результаты статьи [3].

Предположим, что выполнено следующее условие:

$\left(\mathbf{A}_{\omega}^{2}\right) \quad \omega\left(\cdot, x_{f}\right) \in C^{2}\left(\mathbb{R}^{m}, \mathbb{R}\right)$ для любого $x_{f} \in \mathcal{Y} \quad$ и $\omega(x, \cdot) \in L(\mathcal{Y}, \mu, \mathbb{R})$ для любого $x \in \mathbb{R}^{m}$.

Пусть для некоторого $\theta>0$ функция $f_{0}$ задана равенством $(3)$; при $i=1,2, \ldots$ функции семейства $f_{i} \in C(\mathbb{R},[0,1])$ являются неубывающими и удовлетворяют условию

$$
\forall \varepsilon>0, \quad f_{i} \rightarrow f_{0} \text { равномерно на }(-\infty, \theta-\varepsilon] \cup[\theta+\varepsilon, \infty) \text { при } i \rightarrow \infty .
$$

В случае, если стационарное решение (5) существует и не зависит от переменной микровариации $x_{f}$, оно удовлетворяет следующему уравнению:

$$
u(x)=\int_{\Omega} \int_{\mathcal{Y}} \omega\left(x-y, y_{f}\right) f(u(y)) d y_{f} d y, \quad x \in \Omega .
$$

Следуя идеям статьи [8], определим следующий специфический тип решений, который будем далее исследовать.

Для фиксированного $\theta>0$ будем говорить, что $u \in C^{1}(\Omega, \mathbb{R})$ удовлетворяет $\theta$-условию, если

$(\theta 1)$ множество $\Theta=\{x \in \Omega, \quad u(x)>\theta\}$ непусто и ограничено;

$(\theta 2)$ для любой точки $x$, принадлежащей границе $\mathcal{B}$ множества $\Theta$, выполнено неравенство $u^{\prime}(x) \neq 0$;

( $\theta 3)$ найдутся такие $\sigma>0$ и $r>0$, что $u(x)<\theta-\sigma$ для всех $x \in \Omega \backslash B_{\mathbb{R}^{m}}(\Theta, r)$.

В предположении, что $\Omega=\mathbb{R}^{m}$ и для $f=f_{0}$ существует стационарное решение $U \in C^{1}\left(\mathbb{R}^{m}, \mathbb{R}\right)$ уравнения (6), удовлетворяющее $\theta$-условию, исследуются условия существования стационарных решений $U_{i}$ уравнений (6) для $f=f_{i},=1,2, \ldots$, (т.е., в случае непрерывных функций активации) и условия сходимости этих решений к $U$ при $i \rightarrow \infty$. Следующая теорема дает условия сходимости вышеописанных решений $U_{i}, i=1,2, \ldots$ (при условии, что они существуют) к стационарному решению, отвечающему случаю $f=f_{0}$.

Теорема 2.2. Пусть выполнено условие $\left(\mathbf{A}_{\omega}^{2}\right)$, задано некоторое число $\theta>0$ и функиия $U \in C^{1}\left(\mathbb{R}^{m}, \mathbb{R}\right)$ удовлетворяет $\theta$-условию. Тогда существует такое $\varepsilon>0$, что если для любого достаточно большого $r>0$ при $\Omega=\Omega_{r}$ существуют решения $U_{i} \in B_{C^{1}\left(\Omega_{r}, \mathbb{R}\right)}(U, \varepsilon)$ уравнения (6) 
nри $f=f_{i}$ для всех $i$, бо́льших некоторого номера $\widehat{i}$, то существует решение этого уравнения при $f=f_{0}$, являющееся предельной точкой множества $\left\{U_{i}, i=\widehat{i}+1, \widehat{i}+2, \ldots\right\}$. Более того, если решение (6) при $f=f_{0} u \Omega=\Omega_{r}$ (обозначим его $U_{0}$ ) единственно, то

$$
\left\|U_{i}-U_{0}\right\|_{C^{1}\left(\Omega_{r}, \mathbb{R}\right)} \rightarrow 0
$$

Существование стационарных решений $U_{i}$ уравнения (6) при $i=\widehat{i}+1, \widehat{i}+2, \ldots$ установлено в [3] при помощи теории топологической степени. Там же приведены примеры вычисления топологической степени операторов, порожденных частными случаями уравнения (6) для различных симметричных стационарных пространственно локализованных решений (5) в случаях $\Omega=\mathbb{R}$ и $\Omega=\mathbb{R}^{2}$.

В [6] получены условия существования симметричных стационарных локализованных решений уравнения (1) в случае $\Omega=\mathbb{R}$ и сигмоидальной функции активации, а также предложена схема аппроксимации таких решений соответствующими решениями для случая функции активации типа Хевисайда. Приведем данные результаты.

В случае параметризации функции активации, входящей в $(1)$, некоторым $\lambda \in[0,1]$, стационарное решение уравнения (1) принимает вид:

$$
u(x)=\int_{R} \omega(x-y) f_{\lambda}(u(y)) d y, \quad x \in \mathbb{R}, \quad \lambda \in[0,1] .
$$

Относительно функций, входящих в уравнение (1), предполагается следующее:

$\left(\mathbf{A}_{\omega}^{3}\right) \quad$ функция связи $\omega \in B C(\mathbb{R}, \mathbb{R}) \cap L(\mathbb{R}, \mathbb{R})$ липшицева и

$$
\omega(x)=\omega(-x) \forall x \in \mathbb{R}
$$

$\left(\mathbf{A}_{f}^{3}\right)$ при $\lambda=0$ функция активации $f_{0}$ представлена функцией Хевисайда $(3)$; при любом $\lambda \in(0,1]$ функция $f_{\lambda} \in C(\mathbb{R},[0,1])$ является неубывающей и липшицевой, $f_{\lambda}(u)=0$ для всех $u \in(-\infty, \theta]$, и выполнено следующее условие:

для всех $\varepsilon>0 \quad f_{\lambda} \rightarrow f_{0}$ равномерно на $(-\infty, \theta-\varepsilon] \cup[\theta+\varepsilon, \infty)$ при $\lambda \rightarrow 0$,

для всех $\widehat{\lambda} \in(0,1] \quad f_{\lambda} \rightarrow f_{\widehat{\lambda}}$ равномерно на $\mathbb{R}$ при $\lambda \rightarrow \widehat{\lambda}$.

Введем следующие понятия. Для некоторых $\theta>0$ и набора чисел $a_{1}<\ldots<a_{2 N}$ назовем регулярным $\left(\theta, a_{1}, \ldots, a_{2 N}\right)$-бампом такое стационарное решение $U$ уравнения $(1)$, что множество $\left\{a_{1}, \ldots, a_{2 N}\right\}$ является множеством всех решений уравнения $U(x)=\theta, U^{\prime}\left(a_{i}\right) \neq 0$, и существуют такие $\sigma>0$ и $r>0$, что $U(x)<\theta-\sigma$ для всех $x \in \mathbb{R} \backslash B_{\mathbb{R}}\left(\left[a_{1}, a_{2 N}\right], r\right)$.

Далее, для любых $\varepsilon>0$, регулярного $\left(\theta, a_{1}, \ldots, a_{2 N}\right)$-бампа $U$ и чисел $d_{1}, d_{2} \in \mathbb{R}$, удовлетворяющих условию $d_{1}<a_{1}<a_{2 N}<d_{2}$, определим в $C(\mathbb{R}, \mathbb{R})$ множество

$$
K_{\varepsilon}\left(U, d_{1}, d_{2}\right)=\left\{u \in B_{C(\mathbb{R}, \mathbb{R})}(U, \varepsilon), \max _{x \in\left[d_{1}, d_{2}\right]}\left|u^{\prime}(x)-U^{\prime}(x)\right|<\varepsilon\right\} .
$$

Используя теорему о неявной функции и теорию пространств Гельдера, можно доказать следующую теорему.

Теорема 2.3 (см. [6]). Пусть выполнень условия $\left(\mathbf{A}_{\omega}^{3}\right)$ и $\left(\mathbf{A}_{f}^{3}\right)$ и задан регулярнъй (симметричный $)\left(\theta,-a_{N}, \ldots,-a_{1}, a_{1}, \ldots, a_{N}\right)$-бамп $U_{0}$. Пусть такэсе матрица $A=\left(a_{i j}\right)$ с элементами

$$
\begin{gathered}
a_{i i}=\left|U_{0}^{\prime}\left(a_{i}\right)\right|-\omega(0)-\omega\left(2 a_{i}\right), \\
a_{i j}=(-1)^{i+j+1}-\omega\left(a_{i}-a_{j}\right)+\omega\left(a_{i}+a_{j}\right), \quad i \neq j, \quad i, j=1 \ldots, N,
\end{gathered}
$$

имеет обратную. Тогда существует такое $\varepsilon>0$, что при любом $d>a_{N}$ уравнение (7) при каждом $\lambda \in(0,1]$ имеет решение $U_{\lambda}$ на множестве $K_{\varepsilon}\left(U_{0},-d, d\right)$, являющееся регулярным симметричным бампом, причем $\left\|U_{0}-U_{\lambda}\right\|_{C^{1}([-d, d], \mathbb{R})} \rightarrow 0$ nри $\lambda \rightarrow 0$. 
В [6] также был предложен численный метод построения пространственно локализованного стационарного симметричного решения $U_{\lambda}$ для непрерывной функции активации $f=f_{\lambda}$, $\lambda \in(0,1]$, использующий известное решение $U_{0}$ уравнения $(1)$, отвечающее функции активации $f$ типа Хевисайда.

2.2. Динамика нейронного поля. В [10] было рассмотрено уравнение нейронного поля

$$
\alpha \frac{\partial u(t, x)}{\partial t}=-u(t, x)+\int_{\mathbb{R}^{n}} \omega(x, y) f(u(t, y)) d y, \quad t \geqslant 0, x \in \mathbb{R}^{n}, \alpha>0,
$$

с начальным условием

$$
u(0, x)=u_{0}(x)
$$

Ниже приводятся значимые для данного обзора результаты работы [10].

Зафиксируем $\gamma \in(0,1]$.

$\left(\mathbf{A}_{\omega}^{4}\right)$ Предположим, что функция связи $\omega \in B C\left(\mathbb{R}^{n} \times \mathbb{R}^{n}, \mathbb{R}\right)$ удовлетворяет следующим условиям:

$$
\begin{gathered}
\forall x \in \mathbb{R}^{n} \omega(x, \cdot) \in L\left(\mathbb{R}^{n}, \mathbb{R}\right), \\
\exists C_{\omega}>0 \sup _{x \in \mathbb{R}^{n}}\|\omega(x, \cdot)\|_{L\left(\mathbb{R}^{n}, \mathbb{R}\right)}+\sup _{\substack{\forall x, \widehat{x} \in \mathbb{R}^{n} \\
x \neq \widehat{x}}} \int_{\mathbb{R}^{n}} \frac{\omega(x, y)-\omega(\widehat{x}, y)}{|x-\widehat{x}|^{\gamma}} d y \leqslant C_{\omega},
\end{gathered}
$$

Функция $f_{0}$ представлена функцией Хевисайда (3); при $i=1,2, \ldots$ функции семейства $f_{i} \in C(\mathbb{R},[0,1])$ являются неубывающими и удовлетворяют условиям $f_{i}(u)=0$ при $u<\theta-1 / i$, $f_{i}(u)=1$ при $u \geqslant \theta$.

Рассмотрим следующее эквивалентное представление задачи (8), (9) при $f=f_{i}, i=0,1, \ldots$, в виде семейства операторных уравнений:

$$
u(t, x)=u_{0}(x)+\left(\mathcal{F}_{i} u\right)(t, x), \quad t \geqslant 0, \quad x \in \mathbb{R}^{n},
$$

где при каждом $i=0,1,2, \ldots$ оператор $\mathcal{F}_{i}: B C\left([0, \infty) \times \mathbb{R}^{n}, \mathbb{R}\right) \rightarrow B C\left([0, \infty) \times \mathbb{R}^{n}, \mathbb{R}\right)$ задается равенством

$$
\left(\mathcal{F}_{i} u\right)(t, x)=\int_{0}^{t} \frac{1}{\alpha}\left(-u(s, x)+\int_{\mathbb{R}^{n}} \omega(x, y) f_{i}(u(s, y)) d y\right) d s, \quad t \geqslant 0, \quad x \in \mathbb{R}^{n} .
$$

Используя теорему Банаха о неподвижной точке сжимающего оператора и компактность вложения пространства Гельдера (с показателем $\gamma$ ) в пространство ограниченных непрерывных функций, в [10] было получено следующее утверждение.

Теорема 2.4. Пусть выполнено условие $\left(\mathbf{A}_{\omega}^{4}\right)$. Тогда для $f=f_{i}$ при каждом $i=1,2, \ldots$ задача (8), (9) имеет единственное решение $u_{i} \in B C\left([0, \infty) \times \mathbb{R}^{n}, \mathbb{R}\right)$. Если предельнал точка $\widehat{u}$ последовательности $\left\{u_{i}\right\}, i=1,2, \ldots$, решений уравнений (10) удовлетворяет условию

$$
\operatorname{mes}\left(\left\{(t, x) \in[0, \infty) \times \mathbb{R}^{n}, \widehat{u}(t, x)=\theta\right\}\right)=0,
$$

то задача (8), (9) имеет решение при $f=f_{0}$.

Следующий результат, непосредственно вытекающий из доказательства данной теоремы, но не сформулированный в [10] в явном виде, представляет интерес для настоящей работы.

Следствие 2.3. Пусть выполнены условия теоремы 2.4. Если точка сгущения $\widehat{u}=\widehat{u}(t, x)$ последовательности решений уравнений (10) удовлетворяет условию (11), то она является решением задачи (8), (9) при $f=f_{0}$. 
Здесь предлагается следующее обобщение уравнения (1):

$$
u(t, x)=\int_{0}^{t} \int_{\Omega} W(t, s, x, y) f(u(s-\tau(x, y), y)) d y d s+I(t, x), \quad t \in[0, \infty), \quad x \in \Omega,
$$

учитывающее внешнее воздействие, а также эффекты памяти и запаздывания в нейронном поле.

Наличие запаздывания подразумевает рассмотрение данного уравнения вместе с предысторией:

$$
u(t, x)=\varphi(t, x), \quad t \in(-\infty, 0), \quad x \in \Omega .
$$

Ниже исследуются вопросы разрешимости и корректной разрешимости задачи (12), (13). Предположим, что выполнены следующие условия:

$\left(\mathbf{A}_{W}^{5}\right) \quad W \in C\left([0, \infty) \times[0, \infty) \times \Omega \times \Omega, \mathbb{R}^{n}\right)$, а также

$$
\begin{array}{ll}
\sup _{(t, x) \in[0, T] \times \Omega}|W(t, \cdot, x, \cdot)| \in L([0, T] \times \Omega, \mathbb{R}) & \text { для всех } T>0, \\
\lim _{|x| \rightarrow \infty} \sup _{y \in \Omega}|W(t, s, x, y)|=0 & \text { для всех } t, s \in[0, \infty) ;
\end{array}
$$

$\left(\mathbf{A}_{\tau}^{5}\right) \quad \tau \in C(\Omega \times \Omega,[0, \infty)) ;$

$\left(\mathbf{A}_{I}^{5}\right) \quad I \in C_{0}\left([0, \infty) \times \Omega, \mathbb{R}^{n}\right)$

$\left(\mathbf{A}_{\varphi}^{5}\right) \quad \varphi \in C\left((-\infty, 0) \times \Omega, \mathbb{R}^{n}\right), \lim _{|(t, x)| \rightarrow \infty} \varphi(t, x)=0$, а также $\lim _{t \rightarrow 0} \varphi(t, x)=I(0, x)$ для всех $x \in \Omega$.

Пусть при $i=0,1, \ldots$ функция $f_{i}: \mathbb{R}^{n} \rightarrow[0,1]^{n}\left([0,1]^{n}=[0,1] \times \ldots \times[0,1]\right)$ имеет компоненты $f_{i}(u)=\left(f_{i}^{1}\left(u^{1}\right), \ldots, f_{i}^{n}\left(u^{n}\right)\right)^{T}$. При $i=0$ компоненты вектор-функции $f_{0}$ имеют вид функции Хевисайда с пороговыми значениями $\theta^{1}, \ldots, \theta^{n}$, т.е.

$$
f_{0}^{j}\left(u^{j}\right)=\left\{\begin{array}{ll}
0, & u^{j} \leqslant \theta^{j}, \\
1, & u^{j}>\theta^{j},
\end{array} \quad j=1, \ldots, n ;\right.
$$

при каждом $i=1,2, \ldots$ компоненты $f_{i}^{j} \in C(\mathbb{R},[0,1])$ функции $f_{i}$ липшицевы и существует такой вектор $h=\left(h^{1}, \ldots, h^{n}\right), h^{j}>0$, что $f_{i}^{j}\left(u^{j}\right)=0$ для любого $i=1,2, \ldots$ и всех таких $u^{j} \in \mathbb{R}$, что $u_{j}<h_{j}, j=1, \ldots, n$. Пусть также для любого $\varepsilon>0$ выполнено условие

$$
\operatorname{mes}\left(\left\{u \in \mathbb{R}^{n}, f_{i}(u) \notin B_{\mathbb{R}^{n}}\left(f_{0}(u), \varepsilon\right)\right\}\right) \rightarrow 0, \quad i \rightarrow \infty .
$$

Если в уравнении (12) выбрана функция активации $f=f_{i}, i=1,2, \ldots$, то $T$-локальным решением задачи $(12),(13)$ будем называть функцию $u_{T} \in C_{0}\left([0, T] \times \Omega, \mathbb{R}^{n}\right)$, удовлетворяющую на $[0, T] \times \Omega$ уравнению (12) и условию (13). Для задачи (12), (13) при $f=f_{i}, i=1,2, \ldots$, под глобалъным решением понимается такая функция $u_{\infty} \in C_{0}\left([0, \infty) \times \Omega, \mathbb{R}^{n}\right)$, что при любом $T>0$ всякое ее сужение $u_{T} \in C_{0}\left([0, T] \times \Omega, \mathbb{R}^{n}\right)$ на $[0, T] \times \Omega$ является $T$-локальным решением данной задачи.

В случае $f=f_{0}$ под обобщенным $T$-локальным решением задачи (12), (13) понимаем функцию $u_{T} \in C_{0}\left([0, T] \times \Omega, \mathbb{R}^{n}\right)$, удовлетворяющую на $[0, T] \times \Omega$ включению

$$
u(t, x) \in \int_{0}^{t} \int_{\Omega} W(t, s, x, y) \mathcal{H}(u(s-\tau(x, y), y)) d y d s+I(t, x), \quad t \in[0, T], \quad x \in \Omega,
$$

с предысторией (13), где компоненты многозначного отображения $\mathcal{H}=\left(\mathcal{H}^{1}, \ldots, \mathcal{H}^{n}\right)$ заданы следующим образом:

$$
\mathcal{H}^{j}\left(u^{j}\right)= \begin{cases}0, & u^{j}<\theta^{j}, \\ {[0,1],} & u^{j}=\theta^{j}, \quad j=1, \ldots, n . \\ 1, & u^{j}>\theta^{j},\end{cases}
$$

При $f=f_{0}$ будем считать обобщенным глобальным решением задачи (12), (13) функцию $u_{\infty} \in$ $C_{0}\left([0, \infty) \times \Omega, \mathbb{R}^{n}\right)$, сужение которой $u_{T} \in C_{0}\left([0, T] \times \Omega, \mathbb{R}^{n}\right)$ на $[0, T] \times \Omega$ при любом $T>0$ является обобщенным $T$-локальным решением данной задачи (12), (13). 
Теорема 2.5. Пустъ выполнены условия $\left(\mathbf{A}_{W}^{5}\right),\left(\mathbf{A}_{\tau}^{5}\right),\left(\mathbf{A}_{I}^{5}\right),\left(\mathbf{A}_{\varphi}^{5}\right)$. Тогда

(1) для $f=f_{0}$ при любом $T>0$ задача (12), (13) имеет обобщенное $T$-локальное решение, всякое обобщенное Т-локальное решение может быть продолжено до обобщенного глобального решения;

(2) для $f=f_{i}$ при любых $T>0$ и $i=1,2, \ldots$ задача (12), (13) имеет единственное $T$-локальное решение $u_{i T} \in C_{0}\left([0, T] \times \Omega, \mathbb{R}^{n}\right)$, всякое $T$-локальное решение может быть продолжено до единственного глобального решения $u_{i \infty} \in C_{0}\left([0, \infty) \times \Omega, \mathbb{R}^{n}\right)$, последовательность $\left\{u_{i \infty}\right\}$, $i=1,2, \ldots$, компактна в $C_{0}\left([0, \infty) \times \Omega, \mathbb{R}^{n}\right)$.

Если дополнительно для некоторой предельной точки $\widehat{u}_{\infty}$ последовательности $\left\{u_{i \infty}\right\}, i=$ $1,2, \ldots$, выполнено условие

$$
\sup _{x \in \Omega} \operatorname{mes}\left(\left\{(s, y) \in[0, \infty) \times \Omega, \widehat{u}_{\infty}(s-\tau(x, y), y)=\theta\right\}\right)=0
$$

то $\widehat{u}_{\infty}$ является обобщенным глобальным решением задачи (12), (13) при $f=f_{0}$. Если при $f=f_{0}$ решение (12), (13) (скажем, $u_{0 \infty}$ ), единственно, то $u_{i \infty} \rightarrow u_{0 \infty}$ при $i \rightarrow \infty$.

3. Обсуждение. До публикации работы [7] в литературе использовались два следующих способа построения стационарных локализованных решений уравнения (1) для случая непрерывной функции активации: первый способ основывался на методе сингулярного возмущения (см. [9]), второй - на преобразовании (1) в систему нелинейных дифференциальных уравнений высшего порядка, представимую далее в виде гамильтоновой системы, гомоклиническими траекториями которой являлись искомые решения (1) (см. [5]). Первый способ построения является довольно громоздким, и, кроме того, может применяться только в случае симметричной функции связи, имеющей ровно один положительный нуль. Недостатком второго способа также является ограничение выбора функции связи $\omega$ : ее преобразование Фурье должно допускать представление в виде вещественной рациональной функции. В [7] были предложены два естественных итерационных метода построения стационарного локализованного решения, отвечающего непрерывной функции активации, на основе знания о существовании такого решения уравнения (1) с функцией активации типа Хевисайда, а также была показана сходимость этих методов в пространстве непрерывных функций. В [3] был использован подход к проблеме исследования близости стационарных локализованных решений (1) с разрывной и непрерывной функциями активации, основанный на совокупной компактности интегральных операторов Гаммерштейна, порождаемых правой частью уравнения (6), в специальной топологии. Данное свойство позволяет исследовать близость решений при условии их существования, для доказательства которого используется теория топологической степени. Комбинация этих методов позволяет решать рассматриваемую задачу в довольно общих случаях. Главным ограничением применимости аппарата, предложенного в [3], является возможность вычисления топологической степени интегрального оператора Гаммерштейна, соответствующего случаю разрывной функции $f$, действующего в бесконечномерном пространстве. Для некоторых важных типов решений (6) задача вычисления топологической степени несложно решается путем ее трансляции в конечномерное пространство при помощи удобного гомеоморфизма (см. [3]). Метод исследования близости стационарных локализованных решений уравнения (1) с разрывной и непрерывной функциями активации, основанный на применении теоремы о неявной функции в специально построенном функциональном пространстве (см. [6]), имеет ряд преимуществ по сравнению с предыдущим методом, использующим теорию топологической степени. В отличие от [3], результат статьи [6] гарантирует единственность решений аппроксимирующих уравнений (7) с непрерывной функцией активации, причем без предположения гладкости функции связи, а также дает возможность их численного построения. Заметим здесь, что подход работы [7] опирается на теорию монотонных операторов в упорядоченных пространствах и, следовательно, не требует непрерывности оператора Гаммерштейна, для достижения которой в $[3,6,8]$ вводились специальные пространства и топологии. 
В [10] при помощи теоремы Банаха о неподвижной точке сжимающего оператора и свойства компактности в пространстве Гельдера ограниченных непрерывных функций была показана разрешимость разрывного уравнения (1) в классе непрерывных функций, удовлетворяющих условию (11). Данное условие представляет собой обобщение $\theta$-условия, использованного в $[3,8]$, на случай зависящего от времени решения. Результат о близости решений (1) с разрывной и непрерывной функциями активации 2.3 в явном виде в [10] сформулирован не был, однако был получен в ходе доказательства теоремы 2.4. Предложенная авторами данного обзора теорема 2.5, полученная на основе применения теории абстрактных включений Вольтерры к обобщению (12) уравнения (1), учитывающему эффекты запаздывания и памяти, обобщает, в свою очередь, теорему 2.4 и следствие 2.3 п. 2.2 раздела 2. При этом условие (11) является частным случаем использованного в теореме 2.5 условия 15.

В качестве ближайших перспектив исследований по теме настоящего обзора авторы рассматривают следующие направления.

1. Исследование решений уравнений нейронного поля (1) и (12), не удовлетворяющих условиям 11 и (15), соответственно. Такие решения могут возникать, например, при математическом описании различных видов патологической активности в коре больших полушарий головного мозга. Так, стационарные решения, описывающие такую электрическую активность, типично неустойчивы по Ляпунову, что имеет место, например, в случае, когда стационарное решение $U(x)$ не удовлетворяют условию $U^{\prime}(x) \neq 0$ для таких $x$, что $U(x)=\theta$ (см. [8]). Из подобных решений $U(x)$ можно получить зависящие от времени решения типа «движущаяся волна» $U(x+c t)$, где $c$-скорость волны, важное в изучении расстройств нормальной функции мозга (см. [11]). Для этих решений не выполнены ни условие (11), ни условие (15), что влечет нарушение непрерывности соответствующих операторов Гаммерштейна, на которую опираются исследования п. 2.2 раздела 2 данной работы. Однако для изучения решений такого типа представляется возможным разработать аппарат, использующий абстрактные включения Вольтерры в упорядоченных пространствах, который позволит получить результаты о близости вышеописанных решений при переходе от непрерывной к разрывной функции активации.

2. Получение условий сохранения свойства устойчивости по Ляпунову стационарных решений уравнения Amari и его обобщений при переходе от непрерывной к разрывной функции активации. Эти результаты можно будет получить на основе изучения асимптотических свойств спектров соответствующих операторов, которые могут следовать, например, из сходимости производных Фреше данных операторов в некоторой специальной топологии.

\section{СПИСОК ЛИТЕРАТУРЫ}

1. Бурлаков E. О., Жуковский $E$. С. О корректности обобщенных уравнений нейрополей с импульсным управлением// Изв. вузов. Мат. - 2016. - № 5. - Р. 75-79.

2. Amari S. Dynamics of pattern formation in lateral-inhibition type neural fields// Biol. Cybern. - 1977. 27. - P. 77-87.

3. Burlakov E., Ponosov A., Wyller J. Stationary solutions of continuous and discontinuous neural field equations// J. Math. Anal. Appl. - 2016. - 444. - P. 47-68.

4. Burlakov E., Wyller J., Ponosov A. Two-dimensional Amari neural field model with periodic microstructure: Rotationally symmetric bump solutions// Commun. Nonlin. Sci. Numer. Simul. - 2016. - 32. - P. 81-88.

5. Laing C. R., Troy W. C. PDE methods for nonlocal models// SIAM J. Appl. Dynam. Syst. $-2003 .-2$. - P. $487-516$.

6. Oleynik A., Ponosov A., Kostrykin V., Sobolev A. Spatially localized solutions of the Hammerstein equation with sigmoid type of nonlinearity// J. Differ. Equations. - 2016. - 261, № 10. - P. 5844-5874.

7. Oleynik A., Ponosov A., Wyller J. Iterative schemes for bump solutions in neural field model// Differ. Equ. Dynam. Syst. - 2015. - 23, № 1. - P. 79-98.

8. Oleynik A., Ponosov A., Wyller J. On the properties of nonlinear nonlocal operators arising in neural field models// J. Math. Anal. Appl. - 2013. - 398. - P. 335-351.

9. Pinto D. J., Ermentrout G. B. Spatially structured activity in synapticaly couple neuronal networks: II. Lateral inhibition and standing pulses// SIAM J. Appl. Math. - 2001. - 62. - P. 226-243. 
10. Potthast R., Graben P. B. Existence and properties of solutions for neural field equations// Math. Meth. Appl. Sci. - 2010. - 8. - P. 935-949.

11. Ursino M., La Cara G. E. Travelling waves and EEG patterns during epileptic seizure: Analysis with an integrate-and-fire neural network// J. Theor. Biol. - 2006. - 242, № 1. - P. 171-187.

Бурлаков Евгений Олегович

Тюменский государственный университет

E-mail: eb_@bk.ru

Жуковская Татьяна Владимировна

Тамбовский государственный технический университет

E-mail: t_zhukovskaia@mail.ru

Жуковский Евгений Семенович

Тамбовский государственный университет

E-mail: zukovskys@mail.ru

Пучков Николай Петрович

Тамбовский государственный технический университет

E-mail: puchkov@nnn.tstu.ru 